\title{
TEMA-2017: Aspectos básicos del bordaje Integral de las supervivientes de cáncer de mama
}

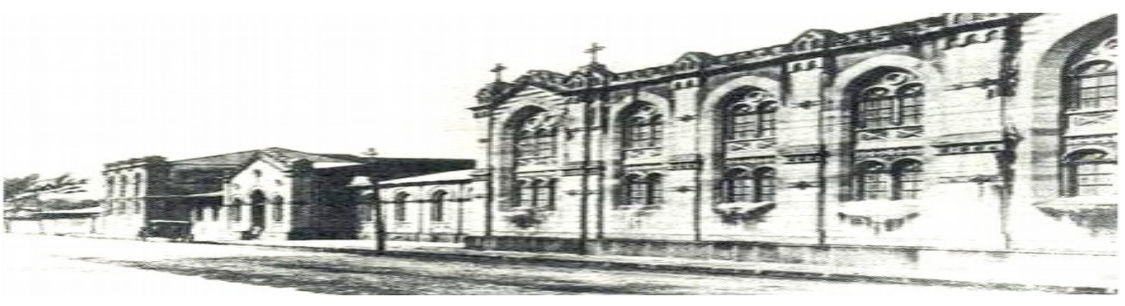

Recibido:

$16 / 12 / 16$

Aceptado:

${ }^{1}$ Jefe de Oncología Médica, Hospital México, San Jose Costa Rica. Profesor adjunto Universidad de Costa Rica. Correo electrónico: denislandaverde@gmail.com

\section{RESUMEN}

Debido a los avances en el manejo multidisciplinario del cáncer se ha incrementado la supervivencia de las pacientes tratadas por cáncer de mama con intención curativa, de la mano con este aumento, también es necesario conocer el abordaje idóneo de las mismas. Este artículo de revisión, pretende mostrar los aspectos relevantes y actualizados en el manejo de este creciente grupo, incluyendo recomendaciones en el tratamiento de comorbilidades asociadas, así como el beneficio potencial de los estilos de vida saludables.

\section{PALABRAS CLAVE}

Supervivientes. Cáncer de mama. Manejo multidisciplinario

\begin{abstract}
The multidisciplinary approach in cancer has incremented the overall survival in breast cancer patients with curative intent, for that reason, it is necessary to know more about the survivorship. The aim of this review is to update of the most relevant aspects in the management and prevention of the most common comorbidities associated in this subset of patients, including the benefits of following recommendations in healthy life styles.
\end{abstract}

\section{KEY WORDS}

Survivorship. Breast Cancer. Multidisciplinary management

INTRODUCCIÓN 
El cáncer de mama actualmente es el tumor maligno más a nivel mundial. Según datos de la Organización Mundial de la Salud, se estima que 508000 mujeres mueren anualmente debido a esta enfermedad, y que según GLOBOCAN 2012, el 58\% de estas muertes ocurren en países menos desarrollados. ${ }^{(1,2)}$ Con respecto a las tasas de supervivencia éstas varían mucho a nivel mundial, con reportes que muestran cifras desde un $80 \%$ en Norteamérica hasta $40 \%$ en países en vías de desarrollo, por ejemplo en América Latina la tasa reportada de supervivencia por cáncer de mama ronda el 70\%. ${ }^{(4)}$ Por lo tanto cada día hay más sobrevivientes de cáncer de mama, por tanto las pacientes, como los médicos, y los sistemas de salud deben conocer este impacto así como las medidas de seguimiento necesarias para abordar este creciente grupo.

En este artículo revisaremos aspectos generales de lo que significa la supervivencia en pacientes con cáncer de mama, así como medidas de seguimiento, estilos de vida saludables y manejo de algunas comorbilidades secundarias a los tratamientos oncológicos.

\section{DISCUSIÓN}

\section{seguimiento}

Definiciones y Modelos de

La definición de sobreviviente o superviviente de cáncer sigue siendo controversial y a la fecha no se ha logrado un acuerdo globalmente aceptado. (5)

Por ejemplo la Coalición Nacional de sobrevivientes del cáncer de los Estados Unidos siglas en ingles NCCS, define a un individuo como superviviente del cáncer desde el momento del diagnóstico mientras su vida esté equilibrada o balanceada. ${ }^{(6)}$

Por otro lado el Instituto Nacional del Cáncer de los Estados Unidos (NCI) considera que supervivencia en cáncer, se enfoca en la salud y la vida de la persona con cáncer después del tratamiento hasta el final de su vida. Esto cubre aspectos físicos, psicológicos y económicos más allá de las fases de diagnóstico y tratamiento.

Esta definición incluye también aspectos relacionados con la habilidad de obtener cuidados en la salud y seguimiento, así como manejo de los efectos a largo plazo de los tratamientos, segundas malignidades y calidad de vida. Los miembros de la familia, amigos y cuidadores son también considerados parte de la experiencia de la supervivencia. ${ }^{(7)}$

Hay muchas más definiciones, pero las mencionadas del NCI y de la NCCS muestran que existe una gran gama de problemas y necesidades más allá de las necesidades físicas o biológicas que que las mismas, deben ser satisfechas tanto en estos pacientes, familia y/o cuidadores así como en el resto de su entorno.

El conocer las definiciones no genera interrogantes en quíen y cómo se debe dar el seguimiento a estas pacientes sobrevivientes, y la literatura no es clara en este aspecto, incluso no hay ningún consenso al respecto.

Se han propuesto varios modelos para abordar el seguimiento, que ya se implementan en diferentes países, los mismos se podrían dividir en aquellos que se ofrecen en centros médicos de la comunidad y los de centros médicos académicos. (8)

Uno de ellos es el modelo de cuidado compartido basado en la comunidad, en este modelo el médico de atención primaria sospecha y/o diagnostica el cáncer, luego lo refiere a un centro de mayor complejidad oncológica donde la paciente es atendida por unos años y luego es devuelta al médico de atención primaria.

Con respecto a los modelos de atención en centros médicos académicos, se han postulado tres: El primero consiste en clínicas consultativas donde se proporciona una única consulta con el oncólogo después de que la paciente terminó su tratamiento y luego se deriva a su centro inicial.

El segundo modelo trata de clínicas de sobrevivientes, en las cuales las pacientes son seguidas por una enfermera entrenada o un médico en el mismo centro de cáncer.

Y el tercer modelo es un programa multidisciplinario de sobrevivientes que tiene colaboración entre los oncólogos, enfermeras entrenadas, trabajadores sociales, psicólogos, etc. Sin embargo parece que todos esos modelos son igual de eficaces, y que incluso enviar a la paciente al médi- 
co de atención primaria tiene los mismos resultados que el continuar con el oncólogo.

Esto basado en varios estudios prospectivos, pero el principal de 968 pacientes con cáncer de mama temprano que se aleatorizaron a ser seguidas por un médico de familia o por un oncólogo, demostró que la tasa de recurrencias, mortalidad, tasas de eventos cínicos serios como fracturas patológicas, compresión del cordón espinal, plexopatía braquial o hipercalcemia fueron equivalentes en ambos grupos. Y aún más la calidad de vida ajustada a salud fue también similar. ${ }^{(9,10)}$

Por lo tanto no existe un modelo único de seguimiento y que cualquiera de los mencionados con anterioridad podrían ser utilizados dependiendo de cada país, centro y situación socio-económica.

En cuanto a Costa Rica, no existe una política clara establecida de este seguimiento a las pacientes, en ciertos hospitales centrales, los cirujanos generales o los cirujanos oncólogos son los que mantienen el control de las pacientes, en otros son los oncólogos médicos. Y rara vez las pacientes son derivadas a su comunidad.

Una vez establecido un programa de seguimiento debe conocerse qué aspectos deben ser evaluados durante el mismo, en el caso específico de las pacientes con cáncer de mama, se debe incluir, vigilancia para detección de recurrencias o nuevos tumores, manejo de efectos a largo plazo de las terapias utilizadas, como ejemplos tenemos el manejo de neuropatía, síntomas de disfunción sexual, dolor, distres psicológico, salud ósea, salud cardiaca, etc.

En los siguientes apartados vamos ir analizando en forma general los diferentes rubros principales que deben ser abordados durante el seguimiento de las sobrevivientes de cáncer de mama.

\section{Generalidades durante el Seguimiento}

Toda paciente tratada con intensión curativa siempre está en riesgo de recurrencia local, regional o sistémica. Por lo que debe tener un plan de seguimiento donde en cada visita debe ser examinada cuidadosamente y obtener una exhaustiva historia clínica buscando síntomas relacionados con el cáncer.
Consensos recientes recomiendan que durante los primeros tres años la paciente debe ser vista cada 3 a 6 meses, luego cada 6 a 12 meses por los 2 siguientes años, y anualmente posterior a este periodo. Sin embargo cada caso puede individualizarse dependiendo de la edad, estadio, riesgo, comorbilidades, etc. ${ }^{(11)}$

Si la paciente está asintomática, el realizar exámenes de imágenes o laboratorio de seguimiento en forma rutinaria no está recomendado, con la excepción de la mamografía anual en caso de mastectomía unilateral, y cirugías conservadoras. La resonancia magnética de mamas de rutina tampoco está recomendada. Desafortunadamente el sobreuso de estos estudios sigue siendo muy frecuente. ${ }^{(12,13)}$

\section{Segundas malignidades}

Además del riesgo de recurrencia se ha descrito la posibilidad de segundas malignidades primarias, muchas veces relacionado a los mismos tratamientos onco-específicos tales como la quimioterapia y/o radioterapia, pero también se ha reportado con el uso de terapias hormonales como por ejemplo el tamoxifeno, así como factores intrínsecos de las pacientes, factores ambiente, genéticos así como interacción genético-ambiental.

Por ejemplo las leucemias secundarias a quimioterapia principalmente debido a agentes alquilantes pueden ocurrir en los primeros 2 a 5 años y el riesgo declina después de los 10 años. Mientras que las malignidades secundarias inducidas por radioterapia aparecen al menos 5 a 10 años después del tratamiento. ${ }^{(14)}$

Lo anterior nos refuerza la necesidad de mantener un control de por vida en estas pacientes y buscar formas para reducir el riesgo de recurrencia o de segundas malignidades.

\section{Medidas que podrían reducir el riesgo de recurrencia}

Los estilos de vida saludables podrían generar una reducción en el riesgo de recurrencia, esto basado principalmente en estudios de índole epidemiológico. Varios ensayos han demostrado que cambios en los estilos de vida, como modificaciones en la dieta, manejo del peso, aumento en 
la actividad física, cese del fumado, entre otras acciones, podrían impactar la sobrevida de estas paciente incrementándola en forma significativa. $(15,16)$

El sobrepeso y la obesidad se han identificado como factores de riesgo para complicaciones postoperatorias, segundos primarios, riesgo de recurrencia entre otros factores. ${ }^{(17)}$

Una revisión sistemática y un meta-análisis que incluyó 12 estudios de cohorte y estudios clínicos con un total de 23832 pacientes que midió el cambio de peso después del diagnóstico de cáncer, demostró que un aumento del peso basal de más de un $10 \%$ incrementa la mortalidad (HR $1.23 ; 95 \%$ IC, $1.09-1.39 ; \mathrm{p}<0.01) .{ }^{(18)}$

En relación al ejercicio no solo mejora la calidad de vida de las pacientes supervivientes de cáncer de mama, sino que también parece disminuir la mortalidad específica por cáncer de mama y mortalidad por otras causas.

Los estudios de actividad física utilizan una medida llamada MET (Intensidad Metabólica en Horas, donde 3 MET equivalen a caminar moderadamente por 2 a 2.9 millas por una hora) por ejemplo el estudio llamado Collaborative Women's Longevity Study (CWLS) que incluyó 4482 mujeres demostró una mejoría en supervivencia general cuando tenían una actividad física mayor o igual a 21 METS por semana. (HR 0.44; $95 \%$ IC $0.32-0.60 ; \mathrm{p}<0.001) .{ }^{(19)}$

Otro estudio longitudinal de 4643 mujeres diagnosticadas con cáncer de mama después que habían entrado en el ensayo conocido como Women's Health Initiative Study mostró que aquellas que después del diagnóstico de cáncer de mama incrementaron o mantuvieron su actividad física de 9 o más MET por semana, alcanzaron una disminución de la mortalidad por todas las causas en forma significativa (HR 0.67; 95\% IC, $0.46-0.96){ }^{(20)}$

Por lo tanto a la luz de toda esta evidencia es aconsejable instar a las pacientes hacer al menos ejercicio de tipo moderado.

\section{¿Y cuál es la recomendación actual sobre la dieta para reducir el riesgo de cáncer?}

Realmente si vemos la evidencia acutal la mimsa es muy limitada, quizá uno de los estudios más importante es el Women's Intervention Nutrition Study (WINS), el cual fue un estudio prospectivo con el fin de reducir en al menos un 15\% la ingesta calórica proveniente de grasas, se reclutó 2437 mujeres con cáncer invasor de mama en estadios tempranos y con una mediana de seguimiento de 60 meses, se demostró que había una reducción en recaídas tanto locales, regionales, a distancia, como contralaterales (HR 0.76; 95\% IC, 0.60-0.98) sin embargo no se encontró diferencias en supervivencia general. Este estudio por lo tanto suguiere que la reducción de la ingesta calórica podría retardar la reaparición del cáncer de mama. ${ }^{(21)}$

Sobre las dietas ricas en vegetales, frutas, fibras y baja en grasas se realizó el estudio Women's Healthy Eating and Living (WHEL), este ensayo aleatorizado realizado en 3088 mujeres con cáncer de mama temprano, no demostró a 7.3 años de media de seguimiento diferencias en recurrencias, nuevos primarios o mortalidad entre el grupo experimental y el control. ${ }^{(22)}$

A la luz de estos dos estudios modificaciones únicas en la dieta no han demostrado impactar la sobrevida general. Sin embargo es recomendable sugerir dietas bajas en grasa porque podría al menos disminuir el riesgo de recurrencias.

vida

Otras Modificaciones en estilos de

Una medida de reducción de riesgo de recurrencia y de otras neoplasias primarias así como mejorar la supervivencia es definitivamente el cese del fumado, hay un meta-análisis reciente que muestra un incremento de aproximadamente un $33 \%$ del riesgo de mortalidad en mujeres que son fumadoras al momento del diagnóstico. ${ }^{(23)}$

Con respecto al consumo de alcohol su efecto después del diagnóstico no está claro. En el estudio LACE donde habían 1897 pacientes con cáncer de mama temprano, se encontró un incremento en el riesgo de recurrencia del cáncer de mama cuando se consumía alcohol (HR 1.35; 95\% IC, 1.00-1.83), pero no se demostró aumento de mortalidad general. ${ }^{(24)}$ 
Otro estudio el WHEL con 3088 pacientes no demostró aumento de recurrencias, y si encontró una mejoría en la mortalidad en el grupo de pacientes que consumían más de $300 \mathrm{~g} / \mathrm{mes}$ de alcohol (HR 0.69; 95\% IC, 0.49-0.97). ${ }^{(25)}$ La recomendación mas reciente de la Sociedad Americana de Oncología Clínica y la Asociación Americana del Cáncer es limitar la ingesta de alcohol y que la misma no sea mayor a una bebida al día. (11)

\section{Salud Ósea en supervivientes del cáncer de mama}

Varios estudios han demostrado que la pérdida de densidad mineral ósea es mayor en las pacientes con cáncer de mama si se compara con las mujeres sin esta enfermedad, se presume que esto es debido a los diferentes tratamientos usados, los mismo van desde manpulación hormonal con glucocorticoides, supresión gonadal, menopausia prematura inducida por quimioterapia, terapia antiestrogénica, etc. ${ }^{(26)}$

Por lo que es recomendación de consensos actuales, hacer a toda mujer posmenopáusica, una densitometría ósea basal y luego cada dos años, y principalmente a las que se encuentran con inhibidores de aromatasa. Y en el caso de mujeres premenopáusicas que estén con supresión ovárica seguir esta misma recomendación. ${ }^{(1)}$

\section{¿Se puede reducir el riesgo de osteoporosis en estas pacientes?}

Para lograr este fin se deben enfocar los esfuerzos en dos grandes vertientes, la primera se refiere a las medidas no famacológicas como modificaciones en los estilos de vida generales, tales como aumentar el ejercicio, disminuir la ingesta etílica, eliminar el tabaquismo. Y la segunda vertiente debe ser orientada a las medidas farmacológicas, dentro de las cuales se puede considerar la suplementación con calcio (aproximadamente $1200 \mathrm{mg}$ diarios) y vitamina D3 (600-1000UI/d) principalmente en mayores de 50 años. ${ }^{(27)}$

Los bifosfonatos se han evaluado para disminuir el riesgo de fracturas osteoporóticas en pacientes recibiendo inhibidores de aromatasa en la adyuvancia en varios estudios entre ellos ABCSG-12, Z-FAST, ZO-FAST, demostrando que el uso de estos agentes incrementan la densidad mineral ósea en la espina lumbar así como en cadera. Igualmente el denosumab un inhibidor del RANK ligando hace un efecto similar. ${ }^{(28,29,30,31,32)}$

El uso adyuvante de los bifosfonatos como tratamiento propiamente para mejorar la sobrevida libre de enfermedad y sobrevida general ha sido evaluado y sigue siendo de gran controversia y debate, puesto que los estudios individuales no logran demostrar en forma categórica que realmente mejoran éstos parámetros. Un meta-análisis de 36 estudios evaluando alrededor de 36000 mujeres demostró que en mujeres con bajos niveles estrogénicos principalmente en pacientes mayores de 55 años, hay una reducción del 34\% en el riesgo de recurrencia ósea $(\mathrm{p}=0.0001)$ y una reducción del $17 \%$ en el riesgo de muerte por cáncer de mama $(\mathrm{p}=0.04) .{ }^{(33)}$

Debido a estos alentadores resultados las asociaciones oncológicas principlamente europeas han recomendado el uso de bifosfonatos adyuvantes sin embargo los casos deben individualizarse conociendo los efectos secundarios y el beneficio potencial de los mismos.

\section{Comorbilidades asociadas a los tratamientos oncológicos}

Como se mencionó anteriormente en las dos últimas décadas se ha aumentado la sobrevida de las pacientes con cáncer de mama pero desafortunamente también se reporta un incremento en la enfermedad cardiovascular (ECV), principalmente en el grupo de los 35 a 44 años.

Los quimioterapéuticos tales como antraciclinas y drogas anti HER2 como trastuzumab han contribuido con la ECV. Pero también la radioterapia ha jugado su papel en la ECV a largo plazo a pesar de mejores técnicas de planificación.

En ese sentido es claro que debe incrementarse el monitoreo de cardiopatías en las pacientes sobrevivientes, y dentro de este control que debe ser cercano monitorizando el perfil lipídico, fomentándo además modificaciones de estilos de vida, y hacer valoración cardiaca activa ante reporte de síntomas importantes como disnea, fatiga, dolor precordial, etc. ${ }^{(11,16,34)}$ 
La neuropatía secundaria a quimioterapia se ha reportado hasta en un $40 \%$ de las pacientes, y puede alterar en forma significativa la calidad de vida de las mismas. No hay un tratamiento claro para esta condición pero en un estudio aleatorizado placebo controlado evaluó el uso de la duloxetina, que mostró un beneficio clínico de un 30 a $50 \%$ en reducción del dolor.

Debido a esto, se podría prescribir duloxetina como una opción, sin embargo varias guías de manejo recomiendan también otros neuromoduladores pero todos tienen niveles bajos a intermedios de evidencia. ${ }^{(35)}$

Con respecto a los síntomas vasomotores de menopausia prematura, se recomienda los inhibidores selectivos de la recaptación de serotonina-norepinefrina o los inhibidores selectivos de la recaptación de serotonina, Gabentina, así como modificaciones de los estilos de vida. Aunque es controversial se prefiere evitar aquellas sustancias que interfieran con la CYP2D6, si se toma conjuntamente tamoxifeno ya que antidepresivos como la paroxetina podrían bajar la conversión del tamoxifeno a sus metabolitos activos. ${ }^{(36,37)}$

No olvidarse de las dimensiones psicosociales y espiritual

Otro aspecto relevante en las pacientes sobrevivientes del cáncer de mama es su estado emocional, se ha reportado altas tasas de depresión, ansiedad, disfunción sexual, fatiga, así como miedo a la recurrencia ${ }^{(38)}$, por lo que hay que indagar en cada visita por estos síntomas para buscar posibles causas y una vez que se descarta alguna condición orgánica subyacente ofrecerle a la paciente consejería, ayuda psicológica, espiritual, psiquiátrica o alguna intervención farmacológica según sea requerida.

\section{CONCLUSIONES}

Cada vez las pacientes que son tratadas por cáncer de mama con intención curativa tienen supervivencias mayores, por lo que deben generarse planes de seguimiento que incluya los aspectos, físicos, médicos, emocionales, espirituales psicológicos, y socio-económicos con el fin de reinsertar a las pacientes a la sociedad para que vuelvan a ser productivas y su calidad de vida sea la más óptima posible.

El personal en salud involucrado en el manejo y seguimiento de las sobrevivientes deben conocer claramente las comorbilidades asociadas al tratamiento así como las recomendaciones generales de estilos de vida para poder actuar en forma oportuna con el fin de mejorar los síntomas de las pacientes, ofrecerles mayor y mejor calidad de vida y aumentar como fin último su supervivencia.

\section{BIBLIOGRAFÍA}

1. Global Health Estimates, WHO 2013, http://www.who.int/cancer/detection/breastc ancer/en/index 1.html

2. Ferlay J, Soerjomataram I, Ervik M, Dikshit $\mathrm{R}$, Eser S, Mathers C, Rebelo M, Parkin DM, Forman D, Bray, F. GLOBOCAN 2012 v1.0, Cancer Incidence and Mortality Worldwide: IARC CancerBase No. 11 [Internet].Lyon, France: International Agency for Research on Cancer; 2013. Available from: http:/globocan.iarc.fr, accessed on 10/08/2016.

3. Coleman MP, et al. Cancer survival in five continents: a worldwidepopulation-based study (CONCORD). Lancet 2008; 9: 730756

4. Justo N. et al. A Review of Breast Cancer Care and Outcomes in Latin America. The Oncologist 2013;18:248-256

5. Dirven L, Van de Poll Franse LV, Aaronson NK, Reijneveld JC. Controversies in defining cancer survivorship. Lancet Oncol 2015; 16(6):610-2.

6. National Coalition for Cancer Survivorship (NCCS). NCCS Mission,2016. http://www.cancercontrol.cancer.gov/aboutus/our-mission. Accessed June 18, 2016

7. National Cancer Institute (NCI) Office of Cancer Survivorship. Definitions, 2016. http://www.cancer.gov/publications/dictionar 
ies/cancer-terms?cdrid $=445089 . \quad$ Accessed June 18, 2016

8. Oeffinger $\mathrm{KC}$, McCabe MS. Models for delivering survivorship care. J Clin Oncol. 2006;24:5117-5124.

9. Grunfeld E, Levine MN, Julian JA, et al. Randomized trial of long-term follow-up for early-stage breast cancer: a comparison of family physician versus specialist care. J Clin Oncol. 2006. 24:848-855.

10. Grunfeld E, Mant D, Yudkin $\mathrm{P}$, et al. Routine follow up of breast cancer in primary care: randomised trial. BMJ. 1996;313:665-669

11. Runowicz CD, Leach CR, Henry NL, et al. American Cancer Society/American Society of Clinical Oncology breast cancer survivorship care guideline. J Clin Oncol. 2016; 34:611-635

12. Salloum RG, Hornbrook MC, Fishman PA, et al. Adherence to surveillance care guidelines after breast and colorectal cancer treatment with curative intent. Cancer 2012; 118 (22):5644-5651

13. Keating NL, Landrum MB, Guadagnoli E, et al. Surveillance testing among survivors of early-stage breast cancer. J Clin Oncol 2007; 25:1074

14. Wood ME, Vogel V, Ng A, et al. Second Malignant neoplasm: assessment and strategies for risk reduction. J Clin Oncolo 2012; $30: 3734$

15. Curtis RE, Freedman DM, Ron E, et al. New Malignancies among Cancer Survivor: Seer Cancer Registries, 1973-2000. NIH Publication No. 05-5302 181-186. Washington, DC: National Cancer Institute; 2006.

16. Bodai BI, Tuso P. Breast Cancer Survivorship: A Comprehensive Review of Long-Term Medical Issues and Lifestyle Recommendations. Perm J. 2015 19(2):4879

17. Ligibel JA, Alfano CM, Corneya KS, et al. American Society of Clinical Oncology position statement on obesity and cáncer. $\mathrm{J}$ Clin Oncol 2014 32:3568-3574

18. Playdon MC, Bracken MD, Sanft TB. et al. Weight gain after breast cancer diagnosis and all-cause mortality: systematic review and meta-analysis. J Natl Cancer Inst. 2015; 107:djv275

19. Holick CN, Newcomb PA, Trentham-Dietz A, et al. Physical activity and survival after diagnosis of invasive breast cancer. Cancer Epidemiol Biomarkers Prev 2008;17:379386

20. Irwin ML, McTiernan A, Manson JE, et al. Physical activity and survival in postmenopausal women with breast cáncer: Results from the women' health initiative. Cancer Prev Res (Phila). 2011;4:522-529

21. Chlebowski RT, Blackburn GL, Thompson CA, et al. Dietary fat reduction and breast cancer outcome: Interim efficacy results from the Women's Intervention Nutrition Study. J Natl Cancer Inst. 2006;98:17671776

22. Pierce JP, Natarajan L, Caan BJ, et al. Influence of a dietary very high in vegetables, fruit, and fiber and low in fat on prognosis following treatment for breast cancer: the Women's Healthy Eating and Living (WHEL) randomized trial. JAMA 2007;298:289-298

23. Berube S, Lemieux J, Moore L, Maunsell E, Brisson J. Smoking at time of diagnosis and breast cancer-specific survival: New findings and systematic review with metaanalysis. Breast Cancer Res. 2014;16:R42

24. Kwan ML, Chen WY, Flatt SW, et al. Postdianosis alcohol consumption and breast cancer prognosis in the after breast cancer pooling project. Cancer Epidemiol Biomarkers Prev. 2013;22:32-41

25. Flatt SW, Thomson CA, Gold EB, et al. Low to moderate alcohol intake is not associated with increased mortality after breast cancer. Cancer Epidemiolol Biomarkers Prev 2010;19:681-688 
26. Gralow JR, Biermann JS, Farooki A, et al. NCCN Task Force Report: Bone Health in Carcer Care. J Natl Compr Canc Netw 2013 11:S1-S50; quiz S51 (suppl 3)

27. Cosman F, de Beur SJ, LeBoff MS, et al. Clinician's guide to prevention and treatment of osteoporosis. Osteroporos Int 2014;25:2359-2381

28. Brufsky AM, Bosserman LD, Caradonna $\mathrm{RR}$, et al. Zoledronic acid effectively prevents aromatase inhibitor-associated bone loss postmenopausal women with early breast cancer receiving adjuvant letrozole: Z-FAST study 36-month follow-up results. Clin Breast Cancer. 2009;9:77-85

29. Brufsky AM, Harker WG, Beck JT, et al. Final 5-year results of Z-FAST trial:adjuvant zoledronic acid maintains bone mass in postmenopausal breast cancer patients receiving letrozole. Cancer. 2012; 118: 1192-1201.

30. Coleman R, de Boer R, Eidtmann $\mathrm{H}$, et al. Zoledronic acid (zoledronate) for postmenopausal women with early breast cancer receiving adjuvant letrozole ( $\mathrm{ZO}$ FAST study): final 60-month results. Ann Oncol. 2013; 24:398-405.

31. Eidtmann $\mathrm{H}$, de Boer R, Bundred N, et al. Efficacy of zoledronic acid in postmenopausal women with early breast cancer receiving adjuvant letrozole: 36month results of the ZO-FAST Study. Ann Oncol. 2010; 21: 2188-2194.

32. Ellis GK, Bone HG, Chlebowski R, et al. Randomized trial of denosumab in patients receiving adjuvant aromatase inhibitors for non metastatic breast cancer. J Clin Oncol. 2008; 26:4875-4882.

33. Coleman R, Powles $\mathrm{T}$, Paterson A, et al; Early Breast Cancer Trialists' Collaborative Group (EBCTCG). Adjuvant bisphosphonate treatment in early breast cancer: metaanalyses of individual patient data from randomised trials. Lancet. 2015;386:13531361.
34. Ganz PA, Hahn EE. Implementing a Survivorship Care Plan for Patients with Breast Cancer. J Clin Oncol 2008;26(5):759-767

35. Smith E, Pang H, Cirrincione C, et al. Effect of duloxetine on pain, function, and quality of life among patients with chemotherapyinduced painful peripheral neuropathy: A randomized clinical trial. JAMA 2013;309:1359-1367

36. Kaplan M, Mahon S, Cope D, et al. Putting evidence into practice: Evidence-based interventions for hot flashes resulting from cancer therapies. Clin J Oncol Nurs 2011;15:149-157

37. Burstein HJ, Prestrud AA, Seidfeld J, et al. American Society of Clinical Oncology clinical practice guideline: Update on adjuvant endocrine therapy for women with hormone receptor-positive breast cancer. $\mathrm{J}$ clin Oncol 2010 28:3784-3796

38. Harrington CB, Hansen JA, Moskowitz M, et al. It's not over when it's over: long-term symptoms in cancer survivor-a systematic review. Int J Psychiatry Med 2010;40:163

\section{CONFLICTO DE INTERÉS Y/O AGRADECIMIENTOS}

El autor declara que no existió ningún conflicto de interés en el presente reporte. 